\title{
Impact of Light Stress on the Synthesis of Both Antioxidants Polyphenols and Carotenoids, as Fast Photoprotective Response in Chlamydomonas reinhardtii: New Prospective for Biotechnological Potential of This Microalga
}

\author{
Cecilia Faraloni $^{1, *}$, Tiziana Di Lorenzo ${ }^{2} \mathbb{D}$ and Alessandra Bonetti ${ }^{2} \mathbb{D}$ \\ 1 National Research Council, Institute of Bio-Economy, CNR, Via Madonna del Piano n.10, Sesto F.no, \\ 50019 Florence, Italy \\ 2 National Research Council, Research Institute on Terrestrial Ecosystems, CNR, Via Madonna del Piano n.10, \\ Sesto F.no, 50019 Florence, Italy; tiziana.dilorenzo@cnr.it (T.D.L.); alessandra.bonetti@cnr.it (A.B.) \\ * Correspondence: cecilia.faraloni@ibe.cnr.it; Tel.: +39-05-5522-5664
}

Citation: Faraloni, C.; Di Lorenzo, T.; Bonetti, A. Impact of Light Stress on the Synthesis of Both Antioxidants Polyphenols and Carotenoids, as Fast Photoprotective Response in

Chlamydomonas reinhardtii: New Prospective for Biotechnological Potential of This Microalga. Symmetry 2021, 13, 2220. https://doi.org/ $10.3390 /$ sym 13112220

Academic Editor:

Alexander Shapovalov

Received: 6 September 2021

Accepted: 15 November 2021

Published: 20 November 2021

Publisher's Note: MDPI stays neutral with regard to jurisdictional claims in published maps and institutional affiliations.

Copyright: (c) 2021 by the authors. Licensee MDPI, Basel, Switzerland. This article is an open access article distributed under the terms and conditions of the Creative Commons Attribution (CC BY) license (https:// creativecommons.org/licenses/by/ $4.0 /)$.

\begin{abstract}
The aim of this study was to investigate the potential role of the microalga Chlamydomonas reinhardtii as an antioxidant source of enriched biomass. This microalga is a model organism deeply investigated for physiological studies, particularly considering carotenoid synthesis in response to stress, to counteract the effects of the formation of free radicals. Less attention has been paid to the profile characterization of other antioxidant compounds, such as polyphenols, which can be synthesized, concomitantly with carotenoids, under photooxidative stress, especially high light. The cultures of $C$. reinhardtii were exposed to three different light intensities, 70, 800 and $1500 \mu$ moles photons $\mathrm{m}^{-2} \mathrm{~s}^{-1}$. The increasing light intensity symmetrically induced the increasing accumulation of both carotenoids and phenolic compounds. The results showed that exposure to high light intensities caused the accumulation of electrons in the electron transport chain, with a reduction in photosynthetic activity. In the same cultures, high light intensity induced the strong increment of polyphenols such as gallic, chlorogenic and coumaric acids, which resulted 6.2-fold, 4-fold and 3.7-fold higher, respectively, than in cells exposed to the lowest intensities. As expected, at the highest light intensity, the strong induction of the xanthophyll cycle and the largest increment of loroxanthin, lutein, $\alpha$-carotene and $\beta$-carotene could be detected. Antioxidant properties doubled with respect to the initial time, both in acetone and methanol cellular extracts of these cultures, revealing a new potential role for biotechnological application of this microalga.
\end{abstract}

Keywords: antioxidants; carotenoids; Chlamydomonas; microalgae; polyphenols; xanthophylls

\section{Introduction}

Interest in the employment of antioxidants from natural sources is increasing considerably, due to the growing market demand for natural compounds and possible difficulties in the utilization of synthetic antioxidants and their possible toxic effects.

Among the founts of natural compounds, microalgae biomass represents a good source of antioxidants, as microalgae are capable of high production of these metabolites (phenolic compounds, carotenoids, polyunsaturated fatty acids) under stress conditions, making increasingly important to focus attention on studying the physiology of these microorganisms [1-3]. Most importantly, microalgae, respect to higher plants, which can be also rich in antioxidant compounds, have a higher growth rate and can be cultivated in photo-bioreactors, in extreme environments, without competing with arable lands.

Under stress conditions - particularly exposure to high light, nutrient limitation, or starvation-the photosynthetic efficiency decreases, with an increase in the reduction level of the electron transport chain. This condition promotes the photo-oxidative stress and 
accumulation of excess energy, which induces photo-protective mechanisms, with the synthesis of antioxidants.

Among these, carotenoids are one of the most studied compounds, as they act both as a light-harvesting complex (LHC) and as photo-protectors. Primary carotenoids, as xanthophylls and B-carotene, are involved in photo-protection through the de-excitation of singlet chlorophyll (Chl), which accumulates in the LHC, especially under high light intensity, a well-studied mechanism in C. reinhardtii [4-6].

Numerous studies have been carried out on the production of carotenoids by different microalgae in order to obtain a large amount of these compounds to be used as food additive, for cosmetic use and medical applications, due to their important role as an antioxidant [7].

Other important compounds present in photosynthetic cells with strong antioxidant properties are phenolic compounds. Polyphenols represent a large and diverse group of chemically ubiquitous substances (plants, fruit and their derivatives, vine, tea, coffee, and microalgae). Due to their capability of chelating metal ions, phenolic compounds are important in the prevention of the formation of radicals, by improving the antioxidant endogenous cell's response [8]. The role of phenolic compounds in counteracting the effect of damage occurring after exposure to high levels of light, is well documented in photosynthetic cells $[9,10]$. Some studies reported polyphenols' synthesis in microalgae during exposure to UV-light, demonstrating an increase in total polyphenols acting as antioxidants [11,12], and other microalgal extracts containing a high phenolic component, resulting in the control of a significant antioxidant activity [13].

Although the role of polyphenols in preventing antioxidant cellular damage is clear, and the ability of microalgae to synthesize phenolic compounds is well known, a deep investigation of polyphenolic production and their characterization in microalgae has not been carried out.

Of particular interest could be the study of the effect of physiological stress on the changes in microalgal biomass composition, concerning the increase of both carotenoids and polyphenols, and their antioxidant properties, in enriched biomass. C. reinhardti is a well-known microalga from the physiological point of view; for this reason, it was considered a very promising candidate for studying the synthesis of these compounds under high light stress.

The aim of this study was to investigate the concomitant induction of carotenoids and polyphenols synthesis in C. reinhardtii, under high light stress. The metabolic response symmetrically induced the increasing accumulation of both carotenoids and phenolic compounds, according to increasing light intensity.

The obtained results may offer the prospect of producing biomass enriched with antioxidants, produced in massive culture.

\section{Materials and Methods}

\subsection{Strains and Culture Conditions}

Culture of the strain of C. reinhardtii CC124 was grown on TAP medium [14]. An amount of $500 \mathrm{~mL}$ of the culture was grown photoetherotrophycally in $500 \mathrm{~mL}$ glass tubes, bubbled with a mixture of air- $\mathrm{CO}_{2}(98 \% v / v)$, at $28^{\circ} \mathrm{C}$, at the light intensity of $70 \mu \mathrm{mol}$ photons $\mathrm{m}^{-2} \mathrm{~s}^{-1}$. When the culture reached the late logarithmic phase, it was used as inoculum for the experiments, at a chlorophyll concentration of $7.18( \pm 0.39) \mathrm{mg} \mathrm{mL}^{-1}$. Culture was exposed on one side to light intensities of $70 \mu \mathrm{mol}$ photons $\mathrm{m}^{-2} \mathrm{~s}^{-1}$ (condition 1: $\mathrm{C} 1$ ), $800 \mu \mathrm{mol}$ photons $\mathrm{m}^{-2} \mathrm{~s}^{-1}$ (condition 2: C2), or $1500 \mu \mathrm{mol}$ photons $\mathrm{m}^{-2} \mathrm{~s}^{-1}$ (condition 3: C3). C1 conditions were chosen because it was the intensity used for the maintenance of the liquid cultures; $\mathrm{C} 2$ was considered as high light intensity for the microalgal cultures, according to the photosynthetic light curve [15]; and C3 was considered as very high light intensity, equivalent to sunlight at the highest intensity [15]. Hereafter, C1, C2 and C3 culture, or extract, will be used to indicate each culture, or its extract, exposed to C1, C2 and C3 conditions, respectively. 
These conditions were maintained for $5 \mathrm{~h}$ to induce a different level of light stress and the induction of synthesis of carotenoids and polyphenols, as photo-protectors. To carry out the experiments, for each light intensity, four separated cultures were used as replicates.

The period of $5 \mathrm{~h}$ was chosen to markedly induce differences among the different culture conditions, without causing cell death.

\subsection{Pigment Extraction}

To extract pigments, $5 \mathrm{~mL}$ of $90 \%$ acetone was, according to the methodology of [16], added to $100 \mathrm{mg}$ of dried biomass (or to the cell pellet of $5 \mathrm{~mL}$ of culture) and vigorously mixed by vortex for $5 \mathrm{~min}$, then the supernatant was collected by centrifugation at $4000 \mathrm{~g}$ for $5 \mathrm{~min}$, and placed in a separated tube. This procedure was repeated on the remaining pellet at least twice, until the pellet resulted as uncolored. The volume was adjusted to $25 \mathrm{~mL}$ in a $25 \mathrm{~mL}$ graduated flask.

\subsection{Analysis of Pigments}

$\mathrm{Chl} \mathrm{a}, \mathrm{Chl} \mathrm{b}$ and total carotenoids were determined spectrophotometrically in $90 \%$ acetone extracts, as previously described [16].

The analysis was performed in triplicate.

\subsection{Determination of Pigments}

On the same extracts, the concentration of individual carotenoids was performed by reversed-phase Beckman System Gold HPLC (module 125 solvent) detector (DAD), model 168 Nouveau, column Luna, C8 (Phenomenex, Torrance, CA, USA), according to [17]. Carotenoids were identified by comparing the retention time and the spectrum with the standards, HPLC grade (Sigma-Aldrich, Louis, MO, USA). Quantification was obtained using the calibration curve made with the relative standard.

The analysis was performed in triplicate.

\subsection{Extraction of Phenolic Compounds}

For phenolic compound extraction, $10 \mathrm{~mL}$ of $100 \%$ methanol was added to $100 \mathrm{mg}$ of dried biomass and sonicated with a probe inserted into the liquid suspension (MSE 100 watt ultrasonic disintegrator, London UK, Cat. N.7100) at the maximum power for $1 \mathrm{~min}$. The tubes containing the samples were put in ice during the sonication. The volume was adjusted to $25 \mathrm{~mL}$ in a $25 \mathrm{~mL}$ graduated flask.

To eliminate the interference of carotenoids and chlorophylls, all extracts were washed by adding petrol $1 / 1 \mathrm{v} / \mathrm{v}$ and centrifuged at $4000 \mathrm{~g}$ for $5 \mathrm{~min}$ to recover the methanol fraction.

\subsection{Determination of Total Polyphenols}

The total phenolic content was determined in the polyphenolic extract, obtained as previously described, by the Folin-Ciocalteau method [18] using gallic acid (Sigma-Aldrich, Louis, MO, USA) as standard.

The analysis was performed in triplicate.

\subsection{Determination of Polyphenolic Compounds}

HPLC-DAD analyses for identification and quantification of the individual phenolic compounds were performed according to [19] on the methanol extracts obtained as previously described. Polyphenols were identified by comparing the retention time and spectrum with the standards, HPLC grade (Sigma-Aldrich, Louis, MO, USA). The quantification was obtained using the calibration curve made with the relative standard.

Analyses of extracts were carried out using a reversed-phase Beckman System Gold HPLC (Brea, CA, USA) (module 125 solvent) detector (DAD), (Brea, CA, USA) model 168 Nouveau. For the separation, a Phenomenex (Torrance, CA, USA) Kinetex PhenylHexyl 100 A $150 \times 4.6 \mathrm{~mm}$ reverse-phase C18 column with identical pre-column operating at $25{ }^{\circ} \mathrm{C}$ was employed. 
The analysis was performed in triplicate.

\subsection{Antiradical Activity}

The analysis was carried out according to [19].

DPPH (2,2-diphenyl-1-picrylhydrazyl) (Sigma-Aldrich, Louis, MO, USA) is a stable radical that, when it is reduced in the presence of an antiradical hydrogen-donor compound, changes its color from violet to yellow. This color change was measured spectrophotometrically, at $517 \mathrm{~nm}$. The extracts were diluted with methanol, as necessary. An amount of $1 \mathrm{~mL}$ of diluted extract was added to $1 \mathrm{~mL}$ of methanol DPPH solution $(63 \mu \mathrm{M})$, mixed and measured immediately, by recording the absorbance at $517 \mathrm{~nm}$. The absorbance was measured again after $20 \mathrm{~min}$. A decrease of $50 \%$ of the initial DPPH concentration was referred to as IC50, which was the inhibition concentration by $50 \%$ of DPPH. Different concentrations of the extracts were used to determine the IC50 $\left(75,50,25,12,5,6.25 \mu \mathrm{g} \mathrm{mL}{ }^{-1}\right)$, diluting the previous solution by 1.5 -fold and then by half. Due to the green color of the acetone extracts, the absorbance value at $517 \mathrm{~nm}$ of $1 \mathrm{~mL}$ of each sample at the same dilution was used for the analysis, added to $1 \mathrm{~mL}$ of methanol, and was subtracted from all the respective absorbance values for each sample. For blank, pure methanol was used.

For each extract, the IC50 was calculated with the following formula:

$$
\% \text { inhibition }=[1-(\mathrm{As}-\mathrm{Ax}) / \mathrm{As}] \times 100
$$

where, As is the initial absorbance of the sample extract in DPPH solution, and Ax is the absorbance of the same sample after $20 \mathrm{~min}$.

The analysis was performed in triplicate.

\subsection{Antioxidant Activity, ORAC Assay (Oxygen Radical Absorbance Capacity)}

The method described by [20] was used, by means of the fluorescence spectrophotometer instrument (Varian Cary Eclipse) (Palo Alto, CA, USA). For the reaction, the mixture consisted of the sample mixed with a free-radical generator (AAPH, 2,2'-azobis (2-aminopropane) dihydrochloride). Fluorescein, used as a target for free radical attack in the mixed solution, encountered conformational changes leading to dose- and timedependent fluorescence quenching. The reagents were added to a quartz cuvette with the following amounts: $2738 \mu \mathrm{L}$ fluorescein $\left(25.5 \mathrm{mg} \mathrm{L}^{-1}\right.$ solution, maintained at $\left.4{ }^{\circ} \mathrm{C}\right)$, $37 \mu \mathrm{L}$ phosphate buffer solution (75 mM, pH 7.4), $150 \mu \mathrm{L}$ Trolox standard, $1 \mu \mathrm{M}$, in final concentration (Sigma-Aldrich, Louis, MO, USA, $20 \mu \mathrm{M}$ ), blank (buffer solution) or $150 \mu \mathrm{L}$ of the sample solution. After $30 \mathrm{~min}$ at $37^{\circ} \mathrm{C}, 75 \mu \mathrm{L}$ of 2,2'-azobis (2-amidino-propane) dihydrochloride (AAPH, $86.8 \mathrm{mg} \mathrm{mL}^{-1}$ in buffer solution and kept in ice) was added and the reaction started. Fluorescence was measured every $5 \mathrm{~min}$ at exciting $\lambda 490 \mathrm{~nm}$ and emission $\lambda 512 \mathrm{~nm}$.

One ORAC unit refers to the value of the area under the curve (AUC), given by the decay of the fluorescence values to the lowest stable value, in the time considered (at least $30 \mathrm{~min})$. The total relative ORAC value of the sample was reported as ORAC unit $(\mu \mathrm{M}) \mathrm{TE}$ (Trolox Equivalents) per mg of sample, given by the following formula:

ORAC value $=\mathrm{k}($ AUCsample - AUCblank $) /($ AUCTrolox - AUCblank $) \times[$ Trolox $] /[$ Sample $]$, with $\mathrm{k}$ the dilution factor; AUC as area under the curve of sample, blank, and Trolox, respectively, in the formula; [Trolox] and [Sample] are the Trolox concentration $(1 \mu \mathrm{mol})$ and the sample concentration (in $\mathrm{mg}$ ).

The analysis was performed in triplicate.

\subsection{Chlorophyll a Fluorescence Transients}

Chlorophyll a fluorescence transients were recorded in $2 \mathrm{~mL}$ of 15 min dark-adapted samples, with a Handy PEA (Hansatech Instruments) under continuous illumination ( $650 \mathrm{~nm}$ peak wavelength, $3500 \mu \mathrm{mol}$ photons $\mathrm{m}^{-2} \mathrm{~s}^{-1}$ light intensity) by light-emitting diodes (LEDs). BiolyzerHP3 software (HPEA/LPA2, Hansatech Instruments, Pentney, UK) was used for the JIP-test [21] to analyze each chlorophyll a fluorescence induction. 
Normalization of the chlorophyll fluorescence data on both $\mathrm{F}_{0}$ and $\mathrm{F}_{\mathrm{m}}$ (basal and maximum fluorescence value, respectively) was utilized, with the transient calculated as relative variable fluorescence $V_{t}=\left(F_{t}-F_{0}\right) /\left(F_{m}-F_{0}\right)$ at all times, with Ft fluorescence at each considered time, to facilitate comparison among the samples [22].

The chlorophyll fluorescence intensity rose from a minimum level (the O level), in less than $1 \mathrm{~s}$, to a maximum level (the P-level) via intermediate steps, labeled as J (2 ms) and I $(30 \mathrm{~ms})$. The rise O-J corresponded to the single turnover reduction of quinone $A\left(Q_{A}\right)$. The rise J-I corresponded to the reduction of the secondary quinone acceptor, quinone $\mathrm{B}$ $\left(\mathrm{Q}_{\mathrm{B}}\right)$, the plasto-quinone (PQ) pool, and the cytochrome b6f complex, at I step to when the partial reoxidation of $\mathrm{QB}$ occurred, when electrons were transferred to Photosystem I (PSI)'s electron acceptor side. Their reduction continued in the next slowest thermal phase (I-P), in less than $1 \mathrm{~s}$ [22].

The following (JIP-test) parameters, calculated from the fluorescence measurements were considered: the initial slope at the beginning of the variable fluorescence, $\mathrm{Mo}=4\left(\mathrm{~F} 300 \mu \mathrm{s}-\mathrm{F}_{0}\right) /\left(\mathrm{F}_{\mathrm{m}}-\mathrm{F}_{0}\right)$, corresponding to the net rate of the reaction center's closure, which increased by trapping and decreased through electron transport; the variable fluorescence at phase $\mathrm{J}, \mathrm{V}_{\mathrm{J}}=\left(\mathrm{F}_{\mathrm{J}}-\mathrm{F}_{0}\right) /\left(\mathrm{F}_{\mathrm{m}}-\mathrm{F}_{0}\right)$, with $\mathrm{FJ}$ the fluorescence value at $\mathrm{J}$ step, indicated the level of $\mathrm{Q}_{\mathrm{A}}$ reduction.

The following flux ratios and parameters were calculated as described by [23]: $\mathrm{F}_{\mathrm{v}} / \mathrm{F}_{\mathrm{m}}$, $\left.\left(\mathrm{F}_{\mathrm{m}}-\mathrm{F}_{0}\right) / \mathrm{F}_{\mathrm{m}}\right)$, the maximum quantum yield of Photosystem II (PSII) for primary photochemistry; $\psi \mathrm{O}=1-\mathrm{V}_{\mathrm{J}}$, the probability that a trapped photon, which triggered the reduction of $\mathrm{Q}_{\mathrm{A}}$ to $\mathrm{Q}_{\mathrm{A}}{ }^{-}$, could move an electron further than $\mathrm{Q}_{\mathrm{A}}{ }^{-}$into the electron transport chain; $\varphi \mathrm{Eo}=\mathrm{F}_{\mathrm{V}} / \mathrm{F}_{\mathrm{m}} \times \psi \mathrm{O}$, the quantum yield of electron transport.

\subsection{Statistical Analysis}

One-way permutational analyses of variance (PERMANOVA) (Anderson et al. 2008) were used to test the differences in the concentrations of carotenoids, chlorophylls, polyphenols, and antioxidants, upon three different treatments (light intensities: 70, 800, and $1500 \mu \mathrm{mol}$ photons $\mathrm{m}^{-2} \mathrm{~s}^{-1}$ ). PERMANOVA was preferred since it has no assumptions to be respected and, therefore, it is convenient for data sets that deviate from normality. The analyses were performed with unrestricted permutations of raw data using Type I of the sum of squares [24]. All PERMANOVAs were run based on Euclidean distance matrices. Pairwise permutational post-hoc $t$ tests were applied when appropriate [24]. Since permutational tests do not require Bonferroni's correction of the $p$-values, the significance was set equal to 0.05 for all tests. When too few $(<100)$ permutations were available for a given test, correct $p$-values were obtained through Monte Carlo random draws from the asymptotic permutation distribution. All statistical analyses were performed using the software E-PRIMER (version 6.1; Primer-E Ltd., Plymouth, UK).

\section{Results}

The results of the main test of PERMANOVA are reported in the Supplementary File (Table S1), while the results of the pair-wise $t$-test are reported in the following paragraphs.

\subsection{Growth}

Changes in chlorophyll, total carotenoids, and biomass content in the cultures exposed to $\mathrm{C} 1, \mathrm{C} 2$, and C 3 conditions are reported in Table 1 . Results showed that concerning the chlorophyll content, the most relevant and significant changes could be detected in C2 culture, as chlorophyll a, chlorophyll b and total chlorophyll concentrations were increased by $84 \%, 69 \%$, and $79 \%$, respectively. Only chlorophyll a differences were not significant. Only small changes, not significant, were observed in C1 and C3 cultures, where variations in the range of $2.2-5.8 \%$ were detected.

Changes in carotenoids were very consistent and significant in C2 and C 3 cultures, increasing by double and 2.37-fold, respectively, compared with the initial time (Table 1), whereas in $\mathrm{C} 1$ culture they only increased by $9 \%$. 
The biomass content was significantly increased (90\%) in C2 and C3 (8\%) cultures and not significantly in C1 (4\%).

Table 1. Changes in chlorophyll (Chl), total carotenoids (Tot carot), and biomass content (DW) for each culture, at the initial

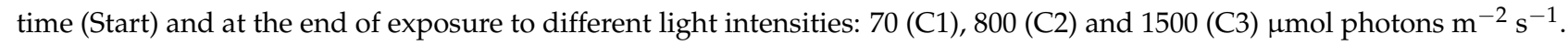
Data are expressed as mean $\pm \mathrm{SD}(n=3)$. For each parameter, significantly different values among culture are marked with different letters (pair-wise permutations $t$-tests, $p<0.05$ ), ${ }^{*}$ indicates non-significant PERMANOVA main test.

\begin{tabular}{cccccc}
\hline Parameter & Unit & Start & C1 & C2 & C3 \\
\hline Chla $^{*}$ & & $4.97( \pm 0.24)$ & $5.08( \pm 0.34)$ & $9.13( \pm 0.49)$ & $5.21( \pm 0.27)$ \\
Chlb & & $2.21( \pm 0.15) \mathrm{a}$ & $2.34( \pm 0.18) \mathrm{a}$ & $3.74( \pm 0.22) \mathrm{b}$ & $2.14( \pm 0.16) \mathrm{a}$ \\
Chla $+\mathrm{b}$ & $\mathrm{mg} \mathrm{L}^{-1}$ & $7.18( \pm 0.39) \mathrm{a}$ & $7.42( \pm 0.52) \mathrm{a}$ & $12.87( \pm 0.71) \mathrm{b}$ & $7.35( \pm 0.43) \mathrm{a}$ \\
Tot carot & & $2.08( \pm 0.13) \mathrm{a}$ & $2.28( \pm 0.19) \mathrm{a}$ & $4.27( \pm 0.17) \mathrm{b}$ & $4.93( \pm 0.15) \mathrm{c}$ \\
DW & & $278( \pm 15.67) \mathrm{a}$ & $290( \pm 11.02) \mathrm{a}$ & $528( \pm 21.10) \mathrm{b}$ & $300( \pm 15.90) \mathrm{a}$ \\
Chla/b & Ratio & $2.25( \pm 0.04) \mathrm{a}$ & $2.17( \pm 0.02) \mathrm{a}$ & $2.44( \pm 0.01) \mathrm{b}$ & $2.43( \pm 0.05) \mathrm{b}$ \\
\hline
\end{tabular}

The statistical analyses indicated that all parameters were significantly different between $\mathrm{C} 2$ and $\mathrm{C} 3$ cultures, except for the Chla/b ratio.

\subsection{Monitoring Stress by Fluorescence Measurements}

Double normalization of the transient curves measured on the cultures exposed to C1, C2, and C3 conditions is reported in Figure 1. The shape of the Kautsky curves clearly reflected the effect of the different light intensities on the photosynthetic apparatus of the cultures. As can be observed, the most relevant changes concerned the different increase at the J step level, due to electron accumulation at the $Q_{A}$ level, which enlarged with the increase of the light intensities.

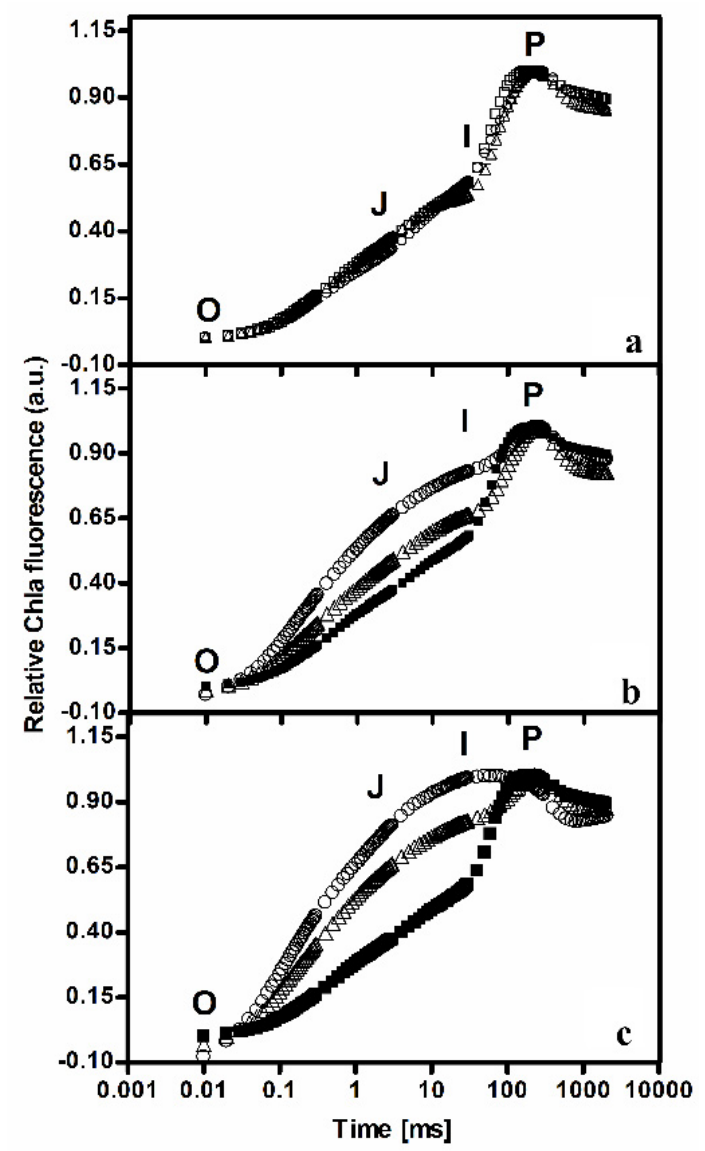

Figure 1. Chlorophyll fluorescence transients of C1 (a), C2 (b) and C3 (c) cultures, during exposure to the light intensities of 70,800 and $1500 \mu \mathrm{mol}$ photons $\mathrm{m}^{-2} \mathrm{~s}^{-1}$, respectively. 
Changes of the JIP-test parameters in the cultures during exposure to the different light conditions are reported in Table 2. It can be observed that values in C1 culture did not significantly change with respect to the initial time. A different situation was observed concerning C2 and C 3 cultures. Statistical analyses indicated that the changes were significantly different for all the cultures for all the other parameters. In particular, in $\mathrm{C} 2$ and $\mathrm{C} 3$ cultures, the $\mathrm{F}_{\mathrm{v}} / \mathrm{F}_{\mathrm{m}}$ was reduced by $54 \%$ and $74 \%$, Mo was more than doubled and tripled, and $\mathrm{V}_{\mathrm{J}}$ was 1.85 -fold and 2.30-fold increased, respectively. Subsequently, due to the electron accumulation at $\mathrm{Q}_{\mathrm{A}}$ level, $\psi \mathrm{O}$ decreased by $40 \%$ and $60 \%$ in $\mathrm{C} 2$ and $\mathrm{C} 3$, respectively. The quantum yield of electron transport, $\varphi$ Eo, decreased by $72 \%$ and $90 \%$, in $\mathrm{C} 2$ and $\mathrm{C} 3$, respectively, due to the effect of exposure to the two high light intensities.

Table 2. JIP-test parameters. The value of each parameter is reported for each culture at the initial time and at the end of exposure to different light intensities: 70 (C1), 800 (C2) and 1500 (C3) $\mu \mathrm{mol}$ photons $\mathrm{m}^{-2} \mathrm{~s}^{-1}$. Data are expressed as mean $\pm \mathrm{SD}(n=3)$. For each parameter, significantly different values among the culture are marked with different letters (pair-wise permutations $t$-tests, $p<0.05$ ).

\begin{tabular}{ccccc}
\hline Parameter & Initial Time & C1 & C2 & C3 \\
\hline $\mathrm{F}_{\mathrm{V}} / \mathrm{F}_{\mathrm{m}}$ & $0.705( \pm 0.003) \mathrm{a}$ & $0.700( \pm 0.009) \mathrm{a}$ & $0.326( \pm 0.001) \mathrm{b}$ & $0.184( \pm 0.008) \mathrm{c}$ \\
$\mathrm{V}_{\mathrm{J}}$ & $0.317( \pm 0.007) \mathrm{a}$ & $0.326( \pm 0.017) \mathrm{a}$ & $0.586( \pm 0.001) \mathrm{b}$ & $0.728( \pm 0.011) \mathrm{c}$ \\
$M_{0}$ & $0.530( \pm 0.019) \mathrm{a}$ & $0.533( \pm 0.022) \mathrm{a}$ & $1.210( \pm 0.009) \mathrm{b}$ & $1.612( \pm 0.013) \mathrm{c}$ \\
$\psi_{\mathrm{O}}$ & $0.683( \pm 0.007) \mathrm{a}$ & $0.674( \pm 0.017) \mathrm{a}$ & $0.414( \pm 0.011) \mathrm{b}$ & $0.272( \pm 0.011) \mathrm{c}$ \\
$\varphi \mathrm{Eo}$ & $0.482( \pm 0.007) \mathrm{a}$ & $0.472( \pm 0.018) \mathrm{a}$ & $0.135( \pm 0.019) \mathrm{b}$ & $0.050( \pm 0.002) \mathrm{c}$ \\
\hline
\end{tabular}

\subsection{Biomass Characterization}

The biomass composition of $\mathrm{C} 1$ culture did not differ from the composition of the cultures used as inoculum (the culture conditions were the same, at a relatively low light intensity), hence the comparison considered the biomass of C1, C2, and C3 cultures, harvested after $5 \mathrm{~h}$ of exposure to the different light intensities, as a result of its response to the different levels of photo-oxidative stress.

The concentrations of the antioxidant compounds-polyphenols and carotenoidsproduced during the exposure to the three different light intensities and the relative antioxidant properties are reported below, in Tables 3 and 4 .

Table 3. Concentrations of polyphenolic compounds in the methanol dried biomass extracts of the cultures exposed to three different light intensities: 70 (C1), 800 (C2) and 1500 (C3) $\mu \mathrm{mol}$ photons $\mathrm{m}^{-2} \mathrm{~s}^{-1}$. Data are expressed as mean $\pm \mathrm{SD}(n=3)$. For each parameter, significantly different values among the culture are marked with different letters (pair-wise permutations $t$-tests, $p<0.05$ ).

\begin{tabular}{cccc}
\hline Polyphenols & C1 & C2 & C3 \\
\hline & & $\mu \mathbf{g ~ g}^{-\mathbf{1}}$ \\
Gallic acid & $0.568 \pm 0.052 \mathrm{a}$ & $1.511 \pm 0.250 \mathrm{~b}$ & $3.516 \pm 0.369 \mathrm{c}$ \\
Chlorogenic acid & $0.200 \pm 0.030 \mathrm{a}$ & $0.476 \pm 0.077 \mathrm{~b}$ & $0.853 \pm 0.216 \mathrm{c}$ \\
Coumaric acid & $0.408 \pm 0.067 \mathrm{a}$ & $0.739 \pm 0.118 \mathrm{~b}$ & $1.517 \pm 0.229 \mathrm{c}$ \\
\hline
\end{tabular}

Table 4. Concentrations of the nine carotenoids in the acetone dried biomass extracts of cultures exposed to three different light intensities: 70 (C1), 800 (C2) and 1500 (C3) $\mu \mathrm{mol}$ photons $\mathrm{m}^{-2}$ s. Data are expressed as mean $\pm \mathrm{SD}(n=3)$. n.d.: not detectable. For each parameter, significantly different values among the culture are marked with different letters (pair-wise permutations $t$-tests, $p<0.05$ ). * indicates non-significant PERMANOVA main test.

\begin{tabular}{cccc}
\hline Carotenoids & C1 & C2 & C3 \\
\hline & mg g $^{-\mathbf{1}}$ \\
Violaxanthin & $1.940 \pm 0.054 \mathrm{a}$ & $1.370 \pm 0.308 \mathrm{~b}$ & $1.620 \pm 0.158 \mathrm{~b}$ \\
Antheraxanthin & $0.468 \pm 0.089 \mathrm{a}$ & $1.028 \pm 0.2384 \mathrm{~b}$ & $1.360 \pm 0.285 \mathrm{~b}$ \\
\hline
\end{tabular}


Table 4. Cont.

\begin{tabular}{cccc}
\hline Carotenoids & C1 & C2 & C3 \\
\hline Zeaxanthin & n.d. & n.d. & $0.976 \pm 0.135$ \\
Loroxanthin & $0.353 \pm 0.078 \mathrm{a}$ & $0.379 \pm 0.122 \mathrm{a}$ & $2.485 \pm 0.505 \mathrm{~b}$ \\
Lutein & $4.618 \pm 0.697 \mathrm{a}$ & $4.037 \pm 1.136 \mathrm{a}$ & $5.785 \pm 0.416 \mathrm{~b}$ \\
Neoxanthin * & $1.320 \pm 0.187$ & $1.159 \pm 0.346$ & $1.054 \pm 0.181$ \\
Cis-lutein * & $1.488 \pm 0.191$ & $1.775 \pm 0.709$ & $1.752 \pm 0.181$ \\
a-carotene & $0.245 \pm 0.041 \mathrm{a}$ & $0.472 \pm 0.115 \mathrm{a}$ & $0.869 \pm 0.193 \mathrm{~b}$ \\
B-carotene & $0.410 \pm 0.066 \mathrm{a}$ & $1.472 \pm 0.432 \mathrm{~b}$ & $1.546 \pm 0.301 \mathrm{~b}$ \\
\hline
\end{tabular}

\subsubsection{Determination of Phenolic Compounds}

Concerning antioxidant metabolites, this study has shown evidence for how the amount of phenolic compounds may increase under high light intensity. The results are reported in Table 3. The total polyphenolic content was $3.07 \mathrm{mg}, 4.44 \mathrm{mg} \mathrm{g}^{-1}$, and $6.5 \mathrm{mg} \mathrm{g}^{-1}$, in $\mathrm{C} 1, \mathrm{C} 2$, and $\mathrm{C} 3$, respectively, indicating the increment induced by the highest light intensity.

The identification of polyphenols by HPLC analysis was carried out for simple phenolic acids using the reference standard. HPLC analysis showed the presence of polyphenols, gallic acid, chlorogenic acid, and coumaric acid. Strong differences could be observed for all the detected compounds; in particular, the highest light intensity corresponded to the highest polyphenolic content. The chromatogram of the HPLC analysis of C3 extract, with the highest concentration of polyphenols, is reported in Figure 2.

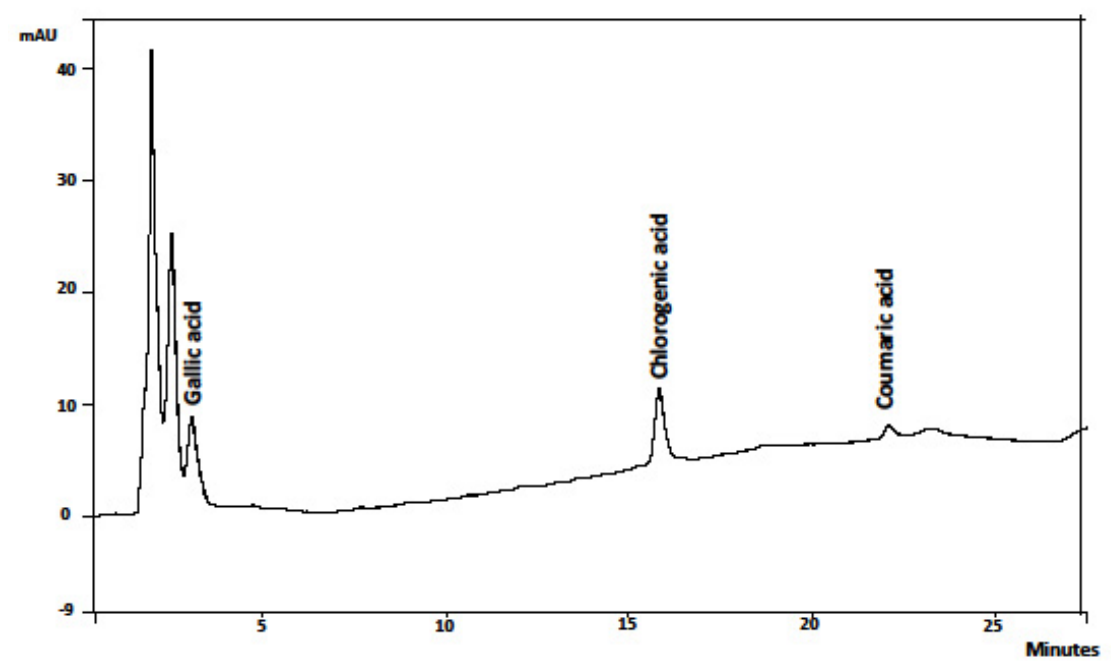

Figure 2. Chromatogram of the polyphenolic extract of C3 culture. For each peak the name of the corresponding phenolic compound (gallic, chlorogenic and coumaric acid, in order according to the retention time) is reported in the chromatogram. The first two peaks in the chromatogram do not correspond to phenolic compounds, but are the results of chromatographic exclusion phenomenon and of the solvent front peak. Both peaks' spectra were checked to confirm that they did not correspond to any phenolic compound.

Gallic acid content under C3 resulted 6-fold and 2-fold higher than under C1 and $\mathrm{C} 2$, respectively, the chlorogenic acid amount was almost 4-fold and 2-fold higher, and coumaric acid 3.7-fold and 2-fold higher than under $\mathrm{C} 1$ and $\mathrm{C} 2$, respectively.

\subsubsection{Determination of Carotenoids and Chlorophylls}

The chromatogram of the HPLC analysis of $\mathrm{C} 3$ extract, with the highest carotenoids concentration, is reported in Figure 3.

The concentrations of the carotenoids in the cultures exposed to the three different treatments (light intensities) are reported in Table 4. 
Differences in the composition of carotenoids in the biomasses evidenced how exposure to C3 induced the highest enrichment in most antioxidant pigments of the group of xanthophylls, loroxanthin, antheraxanthin, zeaxanthin and lutein, and $\alpha$-carotene and $\beta$-carotene. Under $\mathrm{C} 1$ and $\mathrm{C} 2$ conditions the amount of these pigments was significantly different only for violaxanthin, antheraxanthin and $\beta$-carotene, showing the induction of photo-protective response in $\mathrm{C} 2$ and $\mathrm{C} 3$ cultures. In particular, analysis evidenced strong differences in the xanthophyll cycle pigments violaxanthin, antheraxanthin, and zeaxanthin (Table 4) indicating the induction of the xanthophyll cycle under the highest light intensities. Indeed, in C3, an appreciable amount of zeaxanthin could be detected, whereas under $\mathrm{C} 1$ and $\mathrm{C} 2$ conditions it was almost absent. Moreover, antheraxanthin content more than doubled in $\mathrm{C} 2$ and increased by almost 3-fold in $\mathrm{C} 3$ extract, with respect to $\mathrm{C} 1$. Interestingly, the lowest content of violaxanthin was observed in the extracts obtained under both $\mathrm{C} 2$ and C3 conditions, however, in C3 the difference was lower (16\%) than in C2 (29\%), with respect to $\mathrm{C} 1$, probably due to a de-novo synthesis of this pigment.

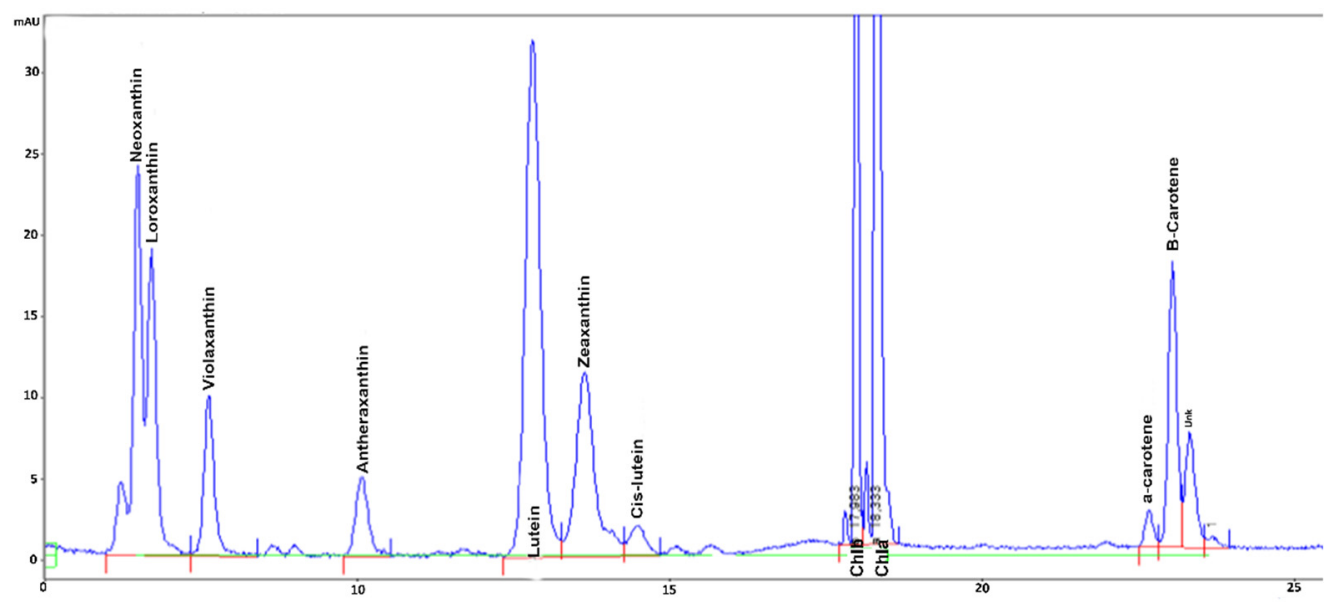

Figure 3. Chromatogram of the carotenoids extract of $\mathrm{C} 3$ culture. For each peak the name of the corresponding carotenoids (neoxanthin, loroxanthin, violaxanthin, antheraxanthin, lutein, zeaxanthin, cis-lutein, chlorophyll b, chlorophyll a, $\alpha$-carotene, $\beta$-carotene, unknown peak in order according to the retention time) is reported in the chromatogram.

This was reflected by the values of the xanthophyll pool concentration, which was $61 \%$ higher in $\mathrm{C} 3$ than in $\mathrm{C} 1$ and $\mathrm{C} 2$ extracts, whereas no difference could be detected between $\mathrm{C} 1$ and C2 (data not shown).

In addition, different changes in the other xanthophylls could be observed. In particular, loroxanthin synthesis exhibited a higher induction, increasing by almost 7-fold in C3 extract, with respect to $\mathrm{C} 1$ and $\mathrm{C} 2$, and the lutein content was incremented by the high light intensity, being $20 \%$ and $30 \%$ higher in C3, than in C1 and C2 extracts, respectively (Table 4). Neoxanthin did not exhibit different values among the extracts, and cis-lutein content was only slightly increased under the highest light intensities, as in C2 and C3 extracts it was $15-16 \%$ higher than in C1.

This trend was confirmed by changes in the other antioxidant carotenoids, $\alpha$-carotene and $\beta$-carotene (Table 4). Indeed, they increased more under the highest light intensities, $\mathrm{C} 2$ and C3, which promoted the synthesis of a doubled and tripled content of $\alpha$-carotene, respectively, and more than tripled the content of $\beta$-carotene, in both $\mathrm{C} 2$ and $\mathrm{C} 3$, with respect to $\mathrm{C} 1$.

The total carotenoids content increase was more consistent under $\mathrm{C} 3$ conditions, more than doubling with respect to $C 1$ and $C 2$, respectively (data not shown).

The concentrations of chlorophyll a, chlorophyll b, total chlorophyll, and chlorophyll $\mathrm{a} / \mathrm{b}$ ratio are reported in Table 5 . Differences among the values were not significant (PERMANOVA main test) for all the cultures. 
Table 5. Concentrations of chlorophyll $a$, chlorophyll $b$, total chlorophyll $(a+b)$ and chlorophyll $a / b$ ratio, in the acetone dried biomass extracts of cultures exposed to three different light intensities. The differences among all the values were non-significant for all the cultures (PERMANOVA main test).

\begin{tabular}{cccc}
\hline Chlorophyll & C1 & C2 & C3 \\
\hline & & $\mathbf{m g ~ g}^{-\mathbf{1}}$ & $17.72 \pm 1.79$ \\
Chla & $16.92 \pm 0.44$ & $17.29 \pm 0.60$ & $7.22 \pm 0.59$ \\
Chlb & $7.55 \pm 0.39$ & $7.09 \pm 0.35$ & $24.95 \pm 2.40$ \\
Chla + b & $24.47 \pm 0.83$ & $24.39 \pm 0.94$ & $2.45 \pm 0.05$ \\
Chla/Chlb & $2.23 \pm 0.06$ & $2.44 \pm 0.04$ & \\
\hline
\end{tabular}

\subsection{Antioxidant Properties}

Both carotenoids and polyphenols were effective to confer antioxidant properties to the biomass of $C$. reinhardtii. Even the extracts with lower carotenoids and polyphenolic content, in the cells exposed to the lower light intensity, revealed that $C$. reinhardtii cells, constitutively, possess considerable antioxidant properties.

Measurements on polyphenolic extracts revealed that the antiradical scavenging property was significantly different among the samples (Table 6). In C3 extract, where the polyphenolic content was more than 4-fold higher than in C1 and C2, the DPPH radical quenching was $45 \%$ higher than in $\mathrm{C} 1$ and $\mathrm{C} 2$. Comparing the antiradical scavenging property of the polyphenolic extracts with that of the standard gallic acid, it was observed that all the extracts exhibited a lower antiradical activity. For both the polyphenolic extracts $\mathrm{C} 1$ and $\mathrm{C} 2$, it was 20-fold less efficient, whereas in C3 it was found to be only 10-fold less efficient. These results fitted well with the respective polyphenolic content in the extract.

Table 6. Antioxidant properties of the methanol extracts of the cultures exposed to three different light intensities: 70 (C1), 800 (C2) and 1500 (C3) $\mu \mathrm{mol}$ photons $\mathrm{m}^{-2} \mathrm{~s}^{-1}$. Data are expressed as mean $\pm \mathrm{SD}(n=3)$. For each parameter, significantly different values among culture are marked with different letters (PERMANOVA test, $p<0.05$ ).

\begin{tabular}{ccc}
\hline & \multicolumn{2}{c}{ Polyphenols Extract } \\
\hline Sample & Antiradical Activity IC $_{\mathbf{5 0}}$ & Antioxidant Activity \\
\hline & $\mu \mathrm{g} \mathrm{ml}^{-1}$ & $\mu \mathrm{mol} \mathrm{TE} \mathrm{\textrm {mg } ^ { - 1 }}$ \\
$\mathrm{C} 1$ & $38.49 \pm 7.85 \mathrm{a}$ & $0.191 \pm 0.023 \mathrm{a}$ \\
$\mathrm{C} 2$ & $38.09 \pm 2.28 \mathrm{~b}$ & $0.198 \pm 0.017 \mathrm{a}$ \\
$\mathrm{C} 3$ & $20.98 \pm 2.48 \mathrm{c}$ & $0.237 \pm 0.009 \mathrm{~b}$ \\
Gallic acid & $2.01 \pm 0.60$ & $26.5 \pm 2.4$ \\
\hline
\end{tabular}

Concerning antioxidant activity, the highest effect was induced under $\mathrm{C} 3$ conditions, followed by C2 and C1. In particular, C3 extract exhibited a value that was $20 \%$ higher than $\mathrm{C} 1$ and $\mathrm{C} 2$, which were very similar. For this extract, the values of the antioxidant activity resulted in a magnitude of 100 -fold lower than standard gallic acid.

Measurements of carotenoids extracts revealed that both the antiradical scavenging property and antioxidant activity were significantly different among the cultures (Table 7).

The high light intensity induced a significant increment of the antiradical activity of the biomass of Chlamydomonas, as the IC50 was reduced by $43 \%$ in the C 3 extract with respect to both $\mathrm{C} 1$ and $\mathrm{C} 2$. The DPPH free radical scavenging activity in C3 also appeared strong in comparison with that of $\alpha$-tocopherol and ascorbic acid (measured with the same method), well-known antioxidant compound, as it was only 4-fold less strong.

In these extracts, the changes of the values of the antioxidant activity followed the same trend as shown in the polyphenolic extracts. Indeed, in C3 extract it was more than doubled, compared with that of $\mathrm{C} 2$ and $\mathrm{C} 1$. $\mathrm{C} 1$ and $\mathrm{C} 2$ were closer to each other, with a small difference of $10 \%$ (Table 7). ORAC activity was compared with that of $\alpha$-tocopherol and ascorbic acid, measured with the same method. In C1 and C2 extracts, the antioxidant activity was less than 2 -fold of the referred standards. It was noteworthy that exposure 
to the highest light intensity induced a stronger increase in the antioxidant activity, as in C3 extract it was even higher, by 39\% and $8 \%$, respectively, than that of $\alpha$-tocopherol and ascorbic acid, respectively.

Table 7. Antioxidant properties of the acetone extracts of cultures exposed to three different light intensities: 70 (C1), 800 (C2) and 1500 (C3) $\mu \mathrm{mol}$ photons $\mathrm{m}^{-2} \mathrm{~s}^{-1}$. Data are expressed as mean \pm SD $(n=3)$. For each parameter, significantly different values among culture are marked with different letters (PERMANOVA test, $p<0.05$ ).

\begin{tabular}{|c|c|c|}
\hline \multirow[b]{2}{*}{ Sample } & \multicolumn{2}{|c|}{ Carotenoids Extract } \\
\hline & Antiradical Activity IC $_{50}$ & Antioxidant Activity \\
\hline & $\mu \mathrm{g} \mathrm{ml}^{-1}$ & $\mu \mathrm{mol}$ TE $\mathrm{mg}^{-1}$ \\
\hline $\mathrm{C} 1$ & $51.30 \pm 3.15 \mathrm{a}$ & $0.376 \pm 0.053 \mathrm{a}$ \\
\hline $\mathrm{C} 2$ & $50.33 \pm 2.03 b$ & $0.405 \pm 0.022 b$ \\
\hline $\mathrm{C} 3$ & $22.29 \pm 0.99 c$ & $0.868 \pm 0.009 c$ \\
\hline$\alpha$-tocopherol & $5.39 \pm 0.35$ & $0.622 \pm 0.03$ \\
\hline Ascorbic acid & $5.55 \pm 0.53$ & $0.802 \pm 0.04$ \\
\hline
\end{tabular}

Very interestingly, the antiradical activity in the polyphenolic extracts of the biomass grown at the lower light intensity showed an IC50 24\% lower than in the carotenoids extract.

Looking at the DPPH radical scavenging capacity, it was relevant that changes of the values in both carotenoid and polyphenolic extracts showed the same behavior, with the antiradical activity of $\mathrm{C} 3$ doubling that of $\mathrm{Cl}$ and $\mathrm{C} 2$ extracts.

The statistical analysis evidenced that values of DPPH in polyphenolic extracts and ORAC in carotenoid extracts were significantly different among the three light intensities. No differences were detected for DPPH and ORAC in carotenoids and polyphenolic extracts, respectively, at 70 and $800 \mu \mathrm{mol}$ photons $\mathrm{m}^{-2} \mathrm{~s}^{-1}$, while significant differences were detected between 70 and $1500 \mu \mathrm{mol}$ photons $\mathrm{m}^{-2} \mathrm{~s}^{-1}$ and between 800 and $1500 \mu \mathrm{mol}$ photons $\mathrm{m}^{-2} \mathrm{~s}^{-1}$.

\section{Discussion}

The unicellular green alga Chlamydomonas reinhardtii is considered a good model organism to study the effect of physiological stress on photosynthetic apparatus and it has been considered particularly interesting for the investigation of the xanthophyll cycle induction $[25,26]$. Moreover, Chlamydomonas can tolerate very high light intensities and it is possible to cultivate this microalga in photobioreactors, outdoor, and under natural light source such as sunlight, and obtain a considerable amount of biomass [27].

The results showed that the changes in chlorophyll fluorescence parameters, during the induction of response to high light exposure, were associated with the fast increase in both polyphenols and carotenoids, acting as antioxidants. This physiological response reflected the symmetry of the two patterns of metabolic reactions involved in carotenoids and polyphenol synthesis under different light intensities (the highest light intensity, the highest carotenoids and polyphenol synthesis induction).

The changes of JIP-test parameters showed that C2 and C3 conditions induced an increment of the reduction level of the photosynthetic apparatus, due to the excess of light energy which led to the accumulation of electrons into the electron transport chain. This was evident also by the increase in $\mathrm{V}_{\mathrm{J}}$, and relative rise of the Kautsky curve at J step level, indicating the electrons were not transported further than $\mathrm{Q}_{\mathrm{A}}$ and the reduction of electron transport rates $(\psi \mathrm{O}, \varphi \mathrm{Eo})[21,25,28]$. The increase in Mo could be due either to this reduction or to the partial damage of the PSII reaction centers, in response to the high light intensities.

For this microalga, the response to exposure to different stress in combination with light stress has been widely investigated particularly in association with the induction of carotenoids synthesis [29-32]. This aspect emerged from the trend of changes of chlorophyll content in the cultures. Under the highest light intensity which was unfavorable for growth, 
C3 culture readily activated photoprotective mechanisms, promoting a high antioxidant carotenoids synthesis and preserving chlorophyll degradation, whereas in C2 cultures the content actually increased. This was reflected by the results observed with exposure to high light, showing the promotion of a protective response in $\mathrm{C} 3$ to the excess of light energy, traduced to the large increase in the most powerful antioxidant carotenoids, such as the xanthophylls loroxanthin, lutein, antheraxanthin, and zeaxanthin, and $\alpha$-carotene and $B$-carotene, which conferred the highest antiradical activity to the extract.

In C2 cultures, the light was strong enough to induce some photo-protective responses such as the synthesis of antioxidant carotenoids which contributed to the prevention of photo-oxidative damage and probably was not necessary to induce chlorophyll content reduction, which actually increased. In $\mathrm{C} 1$ cultures, the light intensity was not strong and cells did not need to reduce chlorophyll content to minimize light absorption, and the intensity was not high enough to strongly increase the chlorophyll content, within $5 \mathrm{~h}$. However, changing the chlorophyll ratio in the cultures exposed to the highest light intensities showed cell response to the stress. Changes of the biomass content followed the same trend as chlorophyll, reflecting the same effect on growth following exposure to different light intensities.

The other important increase in antioxidant compounds found in C. reinhardtii cells, were polyphenols, already reported for this microalga, in relation to antioxidant activity [33]. Nevertheless, a detailed characterization of the phenolic profile for this microalga has not yet been deeply investigated. Moreover, the increase in polyphenols in concomitance to changes in chlorophyll fluorescence, due to photo-oxidative stress, is a remarkable topic. Interestingly, the findings of synthesis of gallic acid in C. reinhardtii cells are in accordance with the results obtained in other microalgae, like Desmodesmus and Nannochloropsis [34], which showed the concentration of gallic acid was in the same range as for $C$. reinhardtii in this study. In addition, the presence of chlorogenic and coumaric acid agree with previous studies, reporting their synthesis in Chlorella vulgaris, Haematococcus pluvialis, Diacronema lutheri, Phaeodactylum sp. [35].

Antioxidant properties in microalgae have been already described [36], particularly considering the contribution of carotenoids [34]. The authors associated the strong contribution of carotenoids, which increased under high light intensity in microalgal biomass, to the increase in the DPPH scavenging properties of extracts of Nannochloropsis, Phaeodactylum, Dunaliella, Chlorella. The results reported here are in accordance with these findings. In particular, the capability of antiradical scavenging of carotenoids in microalgae has been demonstrated for lutein, neoxanthin, zeaxanthin, and $\beta$-carotene $[37,38]$, all incremented pigments in extracts exhibiting the highest antioxidant properties in the present study.

It is interesting to consider that these values, found for carotenoids extracts, were in the same range as those found for the polyphenolic extracts, for $C$. reinhardtii in this study. These results were in line with previous findings, indicating an IC50 in a range of $20-30 \mu \mathrm{g} \mathrm{mL}^{-1}$ for extracts of this microalga, related to the presence of phenolic compounds [33], which was even better than other values found in $C$. reinhardtii methanolic extracts, which reported IC50 of $423.44 \mu \mathrm{g} \mathrm{mL}^{-1}$ [39].

Concerning antioxidant activity, the ORAC values found in both carotenoids and polyphenolic extracts were comparable with those reported for other microalgal strains, ranging around $340-577 \mu \mathrm{mol} \mathrm{TE} \mathrm{g}^{-1}$, for P. tricornutum and D. salina, respectively, among the highest values reported for microalgal extracts [40]. These values could be considered quite high, also considering the comparison with fruit extracts, such as blueberry, $46 \mu \mathrm{mol} \mathrm{TE} \mathrm{g}{ }^{-1}$ [41], and strawberry, $540 \mu \mathrm{mol} \mathrm{TE} \mathrm{g}^{-1}$ [42], which are recognized as good natural sources of antioxidants. In particular, the carotenoid extracts $\mathrm{C} 3$ displayed an antioxidant activity even $50 \%$ higher than those reported for the microalgal strain D. salina and strawberry, and it was even higher than the one found for $\alpha$-tocopherol, and ascorbic acid. The higher content of loroxanthin, neoxanthin, antheraxanthin, $B$-carotene and the presence of zeaxanthin in C3 clearly explained the higher increase in both antiradical and antioxidant activity in this extract of $C$. reinhardtii. Furthermore, the results evidenced the 
involvement of polyphenols in the increment of the antioxidant activity, as the increase in gallic acid, coumaric acid, and chlorogenic acid, under the highest light intensities, concurred with the increment of antioxidant activity in methanolic extracts. These findings were in line with previous studies on edible herbs [43], potato cultivars [44], medical plants [45] and microalga Euglena [46].

This was a relevant indication of the importance of the implementation of polyphenols, in addition to carotenoids, to obtain an antioxidant enriched biomass of C. reinhardtii.

The importance of the employment of this microalga for the synthesis of antioxidant compounds is underlined by the fact that $C$. reinhardtii can acclimate to outdoor conditions, and this is a very interesting capability, which can lead to considering the possibility of producing a large amount of biomass enriched with added-value compounds. The induction of photo-protective responses in an outdoor photobioreactor has already been shown in the strain CC124, which was able to synthesize a considerable content of zeaxanthin during the process of hydrogen production [15]. Although the physiology of C. reinhardtii is well known, the studies carried out for the application of this microalga mainly concerned the improvement of hydrogen production efficiency $[25,27]$, and the changes in the chlorophyll fluorescence for monitoring different kinds of stress, particularly photo-oxidative stress, such as high light exposure and anaerobiosis [28-31]. Some studies have dealt with the use of its exhausted biomass after $\mathrm{H}_{2}$ production, as biochar [47]. Methanolic extracts of C. reinhardtii were found to be cytotoxic against breast cancer, due to the presence of a high phenolic content.

Due to its antioxidant properties, C. reinhardtii has been proposed as dietary supplements as an intermediary for scavenging free radicals, inhibiting human pathogens and an antiapoptotic protein of tumor cells [41].

\section{Conclusions}

Although the optimization of the strategy for microalgal biomass cost-effective production needs further investigation and experimentation, the results reported here are an important indication for obtaining suitable microalgal biomass with the possibility of outdoor cultivation of Chlamydomonas in photobioreactors.

The results of this study indicated that this microalga was able to synthesize a large amount of antioxidants when exposed to the highest light intensity, comparable with sunlight. Although this is a well-known phenomenon reported for microalgae, the study promotes further understanding of the relationship between carotenoids, polyphenols and antioxidant properties.

This study offers new perspectives for the production of antioxidant compounds from a natural source.

Supplementary Materials: The following are available online at https:/ /www.mdpi.com/article/10 .3390 / sym13112220/s1. Table S1: Results of the main test of PERMANOVA: values of the Pseudo-F variables and relative $p$-values. Significant $p$-values are indicated in bold. Pseudo-F's subscripts indicate the degrees of freedom. * indicates car: carotenoids extracts; poly: polyphenolic extracts.

Author Contributions: C.F.: conceptualization, methodology, investigation, writing-review and editing. T.D.L.: software, validation, data curation. A.B.: methodology, formal analysis. All authors have read and agreed to the published version of the manuscript.

Funding: This research did not receive any specific grant from funding agencies in the public, commercial, or not-for-profit sectors.

Data Availability Statement: Not applicable.

Conflicts of Interest: The authors declare no conflict of interest. 


\section{References}

1. Schneider, G.; Figueroa, F.L.; Vega, J.; Chaves, P.; Álvarez-Gómez, F.; Korbee, N.; Bonomi-Baruffi, J. Photoprotection properties of marine photosynthetic organisms grown in high ultraviolet exposure areas: Cosmeceutical applications. Algal Res. 2020, 49, 101956. [CrossRef]

2. Kottuparambila, S.; Thankamonyb, R.L.; Agusti, S. Euglena as a potential natural source of value-added metabolites. A review. Algal Res. 2019, 37, 154-159. [CrossRef]

3. Önem, B.; Doğru, A.; Sevindik, T.O.; Tunca, H. Preliminary study on the effects of heavy metals on the growth and some antioxidant enzymes in Arthrospira platensis-M2 strain. Phycol. Res. 2018, 66, 23-30. [CrossRef]

4. Rees, D.; Noctor, G.D.; Horton, P. The effect of high-energy state excitation quenching on maximum and dark level fluorescence yield. Photosynth. Res. 1990, 25, 199-211. [CrossRef]

5. Niyogi, K.K.; Björkman, O.; Grossman, A. The role of specific xanthophylls in photoprotection. Proc. Natl. Acad. Sci. USA 1997, 94, 14162-14167. [CrossRef]

6. Govindjee, G.; Seufferheld, M.J. Non-photochemical quenching of chlorophyll a fluorescence: Early history and characterization of two xanthophylls-cycle mutants of Chlamydomonas reinhardtii. Funct. Plant Biol. 2002, 29, 1141-1155. [CrossRef]

7. Cerón-García, M.C.; González-López, C.V.; Camacho-Rodríguez, J.; López-Rosales, L.; García-Camacho, F.; Molina-Grima, E. Maximizing carotenoid extraction from microalgae used as food additives and determined by liquid chromatography (HPLC). Food Chem. 2018, 257, 316-324. [CrossRef]

8. Al-Azzawie, H.F.; Mohamed-Saiel, S.A. Hypoglycemic and antioxidant effect of oleuropein in alloxan-diabetic rabbits. Life Sci. 2006, 78, 1371-1377. [CrossRef]

9. Caldwell, M.M.; Robberecht, R.; Flint, S.D. Internal filters: Prospects for UV-acclimation in higher plants. Physiol. Plant. 1983, 58, 445-450. [CrossRef]

10. Dixon, R.A.; Paiva, N.L. Stress-induced phenylpropanoid metabolism. Plant Cell 1995, 7, 1085-1097. [CrossRef]

11. Duval, B.; Shetty, K.; Thomas, W.H. Phenolic compounds and antioxidant properties in the snow alga Chlamydomonas nivalis after exposure to UV light. J. Appl. Phycol. 2000, 11, 559-566. [CrossRef]

12. Kovácik, J.; Klejdus, B.; Backor, M. Physiological responses of Scenedesmus quadricauda (Chlorophyceae) to UV-A and UV-C light. Photochem. Photobioliogy 2010, 86, 612-616. [CrossRef] [PubMed]

13. Hajimahmoodi, M.; Faramazzi, M.A.; Mohammadi, M.; Soltani, N.; Oveisi, M.R.E.; Nafissi-Varcheh, N. Evaluation of antioxidant properties and total phenolic contents of some strains of microalgae. J. Appl. Phycol. 2010, 22, 43-50. [CrossRef]

14. Harris, E. The Chlamydomonas Sourcebook; Trends in Plant Science; Academic Press: San Diego, CA, USA, 1989; Volume 3, pp. 224-230.

15. Scoma, A.; Giannelli, L.; Faraloni, C.; Torzillo, G. Outdoor H2 production in a 50-L tubular photobioreactor by means of a sulfur-deprived culture of the microalga Chlamydomonas reinhardtii. J. Biotechnol. 2012, 157, 620-627. [CrossRef] [PubMed]

16. Lichtenthaler, H.K. Chlorophylls and Carotenoids: Pigments of Photosynthetic Membranes; Packer, L., Douce, R., Eds.; Methods in Enzymology Academic Press: New York, NY, USA, 1987; Volume 148, pp. 350-382.

17. Carneiro, M.; Chini Zittelli, G.; Cicchi, B.; Touloupakis, E.; Faraloni, C.I.; Pereira, H.; Santos, T.F.X.; Otero, A.; Varela, J.; Torzillo, G. Online monitoring of chlorophyll a fluorescence in Nannochloropsis oceanica to assess photochemical changes and the onset of lipid accumulation during nitrogen deprivation. Biotechnol. Bioeng. 2021, 118, 4375-4388. [CrossRef]

18. Folin, O.; Cioaclteau, V. On tyrosine and tryptophane determinations in proteins. J. Biol. Chem. 1927, 73, 627-650. [CrossRef]

19. Romani, A.; Vignolini, P.; Isolani, L.; Ieri, F.; Heimler, D. HPLC-DAD/MS characterization of Flavonoids and hydroxycinnamic derivatives in Turnip tops (Brassica rapa L. subsp. sylvestris L.). J. Agric. Food Chem. 2003, 54, 1342-1346. [CrossRef]

20. Cao, G.; Prior, R.L. Comparison of different analytical methods for assessing total antioxidant capacity of human serum. Clin. Chem. 1998, 44, 1309-1315. [CrossRef]

21. Strasser, R.; Srivastava, A.; Govindge, G. Polyphasic chlorophyll a fluorescence transient in plants and cyanobacteria. Photochem. Photobiol. 1995, 61, 32-42. [CrossRef]

22. Giorio, P.; Sellami, M.H. Polyphasic OKJIP Chlorophyll a Fluorescence Transient in a Landrace and a Commercial Cultivar of Sweet Pepper (Capsicum annuum, L.) under Long-Term Salt Stress. Plants 2021, 10, 887. [CrossRef]

23. Appenroth, K.J.; Stöckel, J.; Srivastava, A.; Strasser, R.J. Multiple effects of chromate on the photosynthetic apparatus of Spirodela plyrhiza as probed by OJIP chlorophyll a fluorescence measurements. Environ. Pollut. 2001, 115, 49-64. [CrossRef]

24. Anderson, M.J. A new method for non-parametric multivariate analysis of variance. Aust. Ecol. 2001, $26,32-46$.

25. Faraloni, C.; Torzillo, G. Phenotypic characterization and hydrogen production in Chlamydomonas reinhardtii QB binding D1 protein mutants under sulfur starvation: Changes in chlorophyll fluorescence and pigment composition. J. Phycol. 2010, 46, 788-799. [CrossRef]

26. Bonente, G.; Pippa, S.; Castellano, S.; Roberto Bassi, R.; Ballottari, M. Acclimation of Chlamydomonas reinhardtii to different growth irradiances. J. Biol. Chem. 2012, 287, 5833-5847. [CrossRef]

27. Torzillo, G.; Scoma, A.; Faraloni, C.; Giannelli, L. Advances in the biotechnology of hydrogen production with the microalga Chlamydomonas reinhardtii. Crit. Rev. Biotechnol. 2015, 36, 485-496. [CrossRef]

28. Faraloni, C.; Torzillo, G. Xanthophyll cycle induction by anaerobic conditions under low light in Chlamydomonas reinhardtii. J. Appl. Phycol. 2013, 25, 1457-1471. [CrossRef] 
29. Bohne, F.; Linden, H. Regulation of carotenoid biosynthesis genes in response to light in Chlamydomonas reinhardtii. Biochim. Biophys. Acta 2002, 1579, 26-34. [CrossRef]

30. Couso, I.; Vila, M.; Vigara, J.; Corsero, B.F.; Vargas, M.Á.; Rodríguez, H.; León, R. Synthesis of carotenoids and regulation of the carotenoid biosynthesis pathway in response to high light stress in the unicellular microalga Chlamydomonas reinhardtii. Eur. J. Phycol. 2012, 47, 223-232. [CrossRef]

31. El-Mekkawi, S.A.; Hussein, H.S.; Abo El-Enin, S.A.; El-Ibinari, N.N. Assessment of stress conditions for carotenoids accumulation in Chlamydomonas reinhardtii as added-value algal products. Bull. Natl. Res. Cent. 2019, 43, 130. [CrossRef]

32. Schüler, L.M.; Santos, T.; Pereira, H.; Duarte, P.; Gangadhar, K.; Florindo, C.; Schulze, P.S.C.; Barreira, L.; Varela, J.C.S. Improved production of lutein and $\beta$-carotene by thermal and light intensity upshifts in the marine microalga Tetraselmis sp. CTP4. Algal Res. 2020, 45, 101732. [CrossRef]

33. Renukadevi, K.P.; Saravana, P.S.; Angayarkanni, J. Antimicrobial and antioxidant activity of Chlamydomonas reinhardtii sp. Int. J. Pharm. Sci. Res. 2011, 2, 1467-1472.

34. Safafar, H.; van Wagenen, J.; Møller, P.; Jacobsen, C. Carotenoids, Phenolic Compounds and Tocopherols Contribute to the Antioxidative Properties of Some Microalgae Species Grown on Industrial Wastewater. Mar. Drugs 2015, 13, 7339-7356. [CrossRef] [PubMed]

35. Goiris, K.; Muylaert, K.; Voorspoels, S.; de Paepe, D.J.E.; Baart, G.; de Cooman, L. Detection of flavonoids in microalgae from different evolutionary lineages. J. Phycol. 2014, 50, 483-492. [CrossRef] [PubMed]

36. Choochote, W.; Suklampoo, L.; Ochaikul, D. Evaluation of antioxidant capacities of green microalgae. J. Appl. Phycol. 2014, 26, 43-48. [CrossRef]

37. Zhang, J.; Sun, Z.; Sun, P.; Chen, T.; Chen, F. Microalgal carotenoids: Beneficial effects and potential inhuman health. Food Funct. 2014, 5, 413-425. [CrossRef]

38. Lee, S.; Lee, J.B.; Lee, K.W.; Jeon, Y.J. Antioxidant properties of tidal pool microalgae, Halochlorococcum porphyrae and Oltamannsiellopsis unicellularis from Jeju Island, Korea. Algae 2010, 25, 45-56. [CrossRef]

39. Jayshree, A.; Jayashree, S.; Thangaraju, N. Chlorella vulgaris and Chlamydomonas reinhardtii: Effective Antioxidant, Antibacterial and Anticancer Mediators. Indian J. Pharm. Sci. 2016, 78, 575-581. [CrossRef]

40. Ahmed, F.; Fanning, K.; Netzel, M.; Turner, W.; Li, Y.; Schenk, P.M. Profiling of carotenoids and antioxidant capacity of microalgae from subtropical coastal and brackish waters. Food Chem. 2014, 165, 300-306. [CrossRef]

41. Prior, R.L.; Cao, G.; Martin, A.; Sofic, E.; McEwen, J.; O’Brien, C.; Lischner, N.; Ehlenfeldt, M.; Kalt, W.; Krewer, G.; et al. Antioxidant capacity as influenced by total phenolic and anthocyanin content, maturity, and variety of Vaccinium species. $J$. Agric. Food Chem. 1998, 46, 2686-2693. [CrossRef]

42. Huang, D.; Ou, B.; Hampsch-Woodill, M.; Flanagan, J.A.; Prior, R.L. High-troughput assay of oxygen radical absorbance capacity (ORAC) using a multichannel liquid handing system coupled with a microplate fluorescence reader in 96-well format. J. Agric. Food Chem. 2002, 50, 4437-4444. [CrossRef]

43. Bordoloi, M.; Bordoloi, P.K.; Dutta, P.; Singh, V.; Nath, S.; Narzari, B.; Bhuian, P.D.; Rao, P.G.; Barua, I.C. Studies on some edible herbs: Antioxidant activity, phenolic content, mineral content and antifungal properties. J. Funct. Foods 2016, 23, 220-229. [CrossRef]

44. Ru, W.; Pang, Y.; Gan, Y.; Liu, Q.; Bao, J. Phenolic compounds and antioxidant activities of potato cultivars with white, yellow, red and purple flesh. Antioxidants 2019, 8, 419. [CrossRef] [PubMed]

45. Tajner-Czopek, A.; Gertchen, M.; Rytel, E.; Kita, A.; Kucharska, A.Z.; Letowska, A.S. Study of antioxidant activity of some medicinal plants having high content of caffeic acid derivates. Antioxidants 2020, 9, 412. [CrossRef] [PubMed]

46. Jerez-Martel, I.; Garcia-Poza, S.; Rodriguez-Martel, G.; Rico, M.; Alfonso-Olivares, C.; Gómez-Pinchetti, J.L. Phenolic profile and antioxidant activity of crude extracts from microalgae and cyanobacteria strains. J. Food Qual. 2017, 2017, 1-8. [CrossRef]

47. Torri, C.; Samorì, C.; Adamiano, A.; Fabbri, D.; Faraloni, C.; Torzillo, G. Preliminary investigation on the production of fuels and bio-char from Chlamydomonas reinhardtii biomass residue after bio-hydrogen production. Bioresourse Technol. 2011, 102, 8707-8713. [CrossRef] [PubMed] 